\title{
Cooperative Surveillance Missions with Multiple Unmanned Ground Vehicles (UGVs)
}

\author{
David A. Anisi, Petter Ögren, Xiaoming Hu and Therese Lindskog
}

\begin{abstract}
This paper proposes an optimization based approach to multi-UGV surveillance. In particular, we formulate both the minimum time- and connectivity constrained surveillance problems, show $\mathcal{N} \mathcal{P}$-hardness of them and propose decomposition techniques that allow us to solve them efficiently in an algorithmic manner.

The minimum time formulation is the following. Given a set of surveillance UGVs and a polyhedral area, find waypointpaths for all UGVs such that every point of the area is visible from a point on a path and such that the time for executing the search in parallel is minimized. Here, the sensor's field of view are assumed to be occluded by the obstacles and limited by a maximal sensor range. The connectivity constrained formulation extends the first by additionally requiring that the information graph induced by the sensors is connected at the time instants when the UGVs stop to perform the surveillance task. The second formulation is relevant to situation when mutual visibility is needed either to transmit the sensor data being gathered, or to protect the team from hostile persons trying to approach the stationary UGVs.
\end{abstract}

\section{INTRODUCTION}

$\mathrm{T}$ he surveillance and security solutions of today are based on a combination of human guards, electronic systems (cameras, intrusion alarms), physical security (fences, gates) and software (verification, logging). However, recent scientific and technological developments enable more autonomous and mobile complementary solutions. It is therefore not surprising that the research area of control of surveillance vehicles is also active and growing. In this paper, we extend our previous work [1], [2], on cooperative surveillance using multiple UGVs in terms of the algorithms and theoretical properties such as formal $\mathcal{N} \mathcal{P}$-hardness proofs.

The main focus of this paper will lie on two different optimization problems, namely the Minimum Time UGV Surveillance Problem (MTUSP) and the Connectivity Constrained UGV Surveillance Problem (CUSP). Informally, the MTUSP is the following. Given a set of surveillance UGVs and a user defined area to be covered, find waypoint-paths such that:

- the area is completely surveyed,

- the time for performing the search is minimized.

Funded by the Swedish defence materiel administration (FMV) and Swedish armed forces through the Technologies for Autonomous and Intelligent Systems (TAIS) project, 297316-LB704859.

D. Anisi, X. Hu and T. Lindskog are with the Institution of Optimization and Systems Theory, Royal Institute of Technology (KTH), SE-100 44, Stockholm, Sweden, $\{$ anisi, hu, tlinds $\} @$ kth.se

P. Ögren is with the Department of Autonomous Systems, Swedish Defence Research Agency (FOI), SE-164 90, Stockholm, Sweden, petter.ogrenefoi.se
The on-board sensor's field of view are assumed to be occluded by the obstacles and limited by a maximal sensor range. In the CUSP formulation, in addition to the above, we also require that

- the information graph is kept recurrently connected, i.e. the UGVs are mutually visible when they stop to perform the surveillance task.

Here connectivity constraints of both line-of-sight and limited sensor range types are considered. More formal statement of the MTUSP and CUSP are provided in Section IV and an illustrative surveillance mission can be seen in Figure 1.

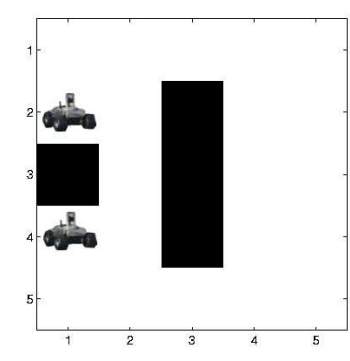

(a)

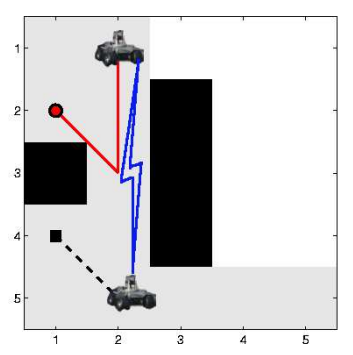

(c)

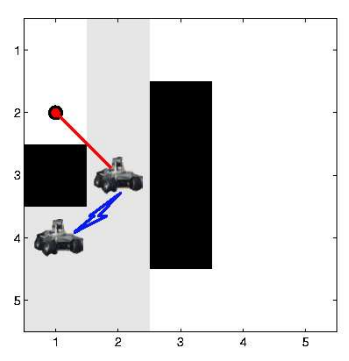

(b)

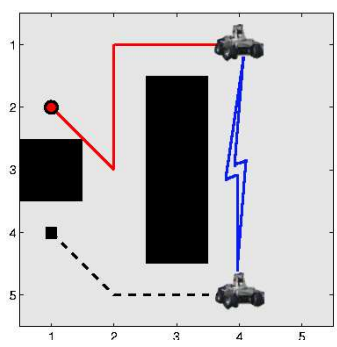

(d)
Fig. 1. Four snapshots during an illustrative minimal CUSP mission. The two UGVs start off to the left, depicted in Figure 1(a), and maintain connectivity recurrently at the three surveillance instances, Figures 1(b)1(d). The area already visited is shadowed and the mission is completed once the entire area is surveyed. Notice how the two UGVs are allowed to split temporarily in order to pass on different sides of the rightmost obstacle.

The remainder of this paper is organized as follows. Section II provides a concise exposition of related work. Section III then presents some basic concepts that underly the rest of the paper. The considered problems are formally defined in Section IV and the proposed algorithms for solving them efficiently can be found in Section V. Simulations illustrating the approach are presented in Section VI. Finally, the paper is concluded in Section VII. 


\section{RELATED WORK}

For the purpose of the MTUSP formulation, we divide the rich set of work in the field of coverage problems into the following three categories: First moving sensor platforms, where the main limitation on field of view is the physical sensor range. Applications where such a formulation is reasonable include vacuum cleaning and demining [3], general coverage [4], multi robot coverage [5] and some robotic security applications [6]. Furthermore, a number of UAV surveillance papers, such as [7], [8] fall into this category. The pursuit-evasion problem, where a number of pursuers try to find an evader is sometimes also formulated in this way [9].

The second category consists of problems dealing with positioning of static sensors where occluding objects and walls present the main limitation on field of view. Such formulations are found in the so called Art Gallery Problems, where the number of guards required to monitor a building is to be minimized (see e.g. [10] and the excellent survey in [11]).

In the third category, where the field of view of moving guards is mainly limited by occluding walls and other objects, we also find results building on the Art Gallery work. In an indoor environment, the pursuit-evasion problem can be solved with a guarantee that a moving evader will be caught [12]. Some of this work also deals with the situation where the area is unknown [13]. The MTUSP falls in this third category, but differ from earlier work in terms of the minimum time objective.

As for literature relevant for the CUSP formulation, a number of publications have been devoted to different aspects of the communication maintenance problem for mobile platforms. The great majority of these papers have focused on the sensor range constraint and often deal with obstaclefree environments. However, [14] and [15] consider communication restrictions involving both limited sensor range and line-of-sight constraints in the presence of obstacles. In the following, both these papers will be discussed in detail.

The problem formulation in [14] considers optimal path planning for a number of relay vehicles that have the mission of maintaining a chain of line-of-sight communication links that connect a given leader vehicle to the ground station. In accordance with their previous work in multi-vehicle path planning, Schouwenaars et al. use binary variables to capture connectivity between subsequent relay vehicles and end up solving a mixed integer linear program (MILP).

The problem considered in [15] is closely related to our work. However, Esposito and Dunbar aim at reaching a final configuration while our high-level objective is to complete the surveillance mission. They furthermore utilize a potential function to synthesize a feasible movement direction for the vehicles and consider the case when a fixed information graph is given a priori and focus on maintaining these given links intact throughout the entire duration of the motion. This is equivalent with maintaining 1-hop connectivity of the information graph.

\section{PRELIMINARIES}

The areas to be searched in this paper are all going to be so called orthogonal polygons with holes (obstacles), thus we denote them $A$, for area. The orthogonality property is however a matter of implementational convenience for the maximal convex cover subproblem. It should be noted that the provided algorithms are not limited to the orthogonal case, but can handle any general polygon-with-holes type of environment.

Definition 1 (Orthogonal polygons with holes): A polygon $Q$ in the plane is an ordered sequence of points $q_{1}, \ldots, q_{n} \in \mathbb{R}^{2}, n \geq 3$, called vertices of $Q$ together with the line segments $q_{i}$ to $q_{i+1}$ and $q_{n}$ to $q_{1}$, called edges. In the following we assume that none of these edges intersect. A polygon is called orthogonal if adjacent edges are orthogonal. Given a polygon $Q$ and a set of $h$ disjoint polygons $Q_{1}, \ldots, Q_{h}$ contained in $Q$ we call the set $A=$ $Q \backslash\left\{Q_{1} \cup \ldots \cup Q_{h}\right\}$ a polygon with $h$ holes.

In order to define our surveillance problems we make the following definitions.

Definition 2 (Guardiance): Given two points $p$ and $q$ in $A$ we say that $p$ is visible from $q$ if the line segment joining $p$ and $q$ is contained in $A$

$$
\alpha p+(1-\alpha) q \in A, \quad \forall \alpha \in\left[\begin{array}{ll}
0 & 1
\end{array}\right]
$$

and the distance between them is not greater than the sensor range,

$$
\|p-q\| \leq R .
$$

A set of points $H=\left\{h_{1}, \ldots, h_{k}\right\} \subset A$ guards $A$ if for all $p \in A$ there exists $h_{i} \in H$ such that $p$ is visible from $h_{i}$.

Definition 3 (Maximal convex cover): A convex cover $C$ of $A$ is a set of convex sets $C=\left\{c_{i}\right\}$ such that $\left|c_{i}\right| \leq R$ and $A \subseteq \cup_{i} c_{i}$. Here, $\left|c_{i}\right|=\sup _{a, b \in c_{i}} \operatorname{dist}(a, b)$ denotes the diameter of the set $c_{i}$. We define a maximal convex cover of $A$ to be a convex cover $C=\left\{c_{i}\right\}$ of $A$, such that for all $i$, there is no convex set $s \subseteq A$ such that $|s| \leq R$ and $s \supset c_{i}$.

Definition 4 (Visiting waypoint path): A waypoint path $P$ is an ordered set of points $P=\left\{p_{1}, \ldots, p_{n}\right\}$. Any convex cover $C$ is said to be visited by the waypoint path $P=$ $\left\{p_{1}, \ldots, p_{n}\right\}$ if $\forall c_{i} \in C \exists p_{j} \in P: p_{j} \in c_{i}$.

Given the above definitions, the following lemma can be stated.

Lemma 1: If there exists a convex cover $C$ of $A$ such that the path $P$ visits $C$, then $P$ guards $A$

Proof. Since $P$ visits $C$, and every set $c_{i}$ in $C$ is convex with $\left|c_{i}\right| \leq R, P$ guards every set $c_{i}$. Furthermore, since $A \subseteq \cup_{i} c_{i}, P$ guards $A$.

Next, two concepts needed for making a formal statement of the CUSP are presented.

Definition 5 (Information graph): Let $\mathcal{V}=\left\{v_{1}, \ldots, v_{N}\right\}$ denote the vertex set representing the $N$ UGVs. There is a link, $e_{i j} \in \mathcal{E}(t)$, in the information graph, $\mathcal{G}(t)=(\mathcal{V}, \mathcal{E}(t))$, if and only if

$$
\left\|p_{i}(t)-p_{j}(t)\right\| \leq R \quad \text { and }
$$




$$
\alpha p_{i}(t)+(1-\alpha) p_{j}(t) \in A, \quad \forall \alpha \in\left[\begin{array}{ll}
0 & 1
\end{array}\right] .
$$

Here $R$ denotes the limited sensor range and $p_{i}(t) \in \mathbb{R}^{2}$ is the position of UGV $i$ at time $t$.

Definition 6 (Connectivity-primitive): A connectivityprimitive $s=\left\{p_{1}, \ldots, p_{N}\right\} \in \mathbb{R}^{2 \times N}$ is a collection of $N$ UGV positions $p_{i} \in \mathbb{R}^{2}$, that induce an information graph that is connected.

The notion of connectivity-primitive is depicted in Figure 3

\section{Problem Formulation}

In this section, using concepts from Section III, we state the two surveillance problems considered in this paper, namely the Minimum Time UGV Surveillance Problem (MTUSP) and the Connectivity Constrained UGV Surveillance Problem (CUSP).

Problem 1 (MTUSP): Given $N$ UGVs and a polyhedral area $A$, find a set of waypoint-paths $P=\left\{P^{1}, \ldots, P^{N}\right\}$ that solve the following optimization problem

$$
\begin{array}{ll}
\min _{P} \max _{i \in \mathbb{Z}_{N}^{+}} & \sum_{j=1}^{n_{i}-1}\left\|p_{j}^{i}-p_{(j+1)}^{i}\right\| \\
\text { such that } & \cup_{i} P^{i} \text { guards } A
\end{array}
$$

Here $P^{i}=\left\{p_{1}^{i}, \ldots, p_{n_{i}}^{i}\right\}$ and the start and finish depots, denoted by $p_{1}^{i}, p_{n_{i}}^{i}, i \in \mathcal{Z}_{N}^{+} \triangleq\{1, \ldots, N\}$, may be given.

Remark 1 (Sensor field of view): In the above problem statement, each point in $A$ is demanded to be visible from some point in $P$. This is reasonable in the case of omnidirectional sensors. It is however also relevant in the case of cameras mounted on pan-tilt units. In these cases the time right before and after passing $p_{j}^{i}$ must be used to cover the areas visible from $p_{j}^{i}$. If necessary, the UGVs will have to slow down to facilitate the sensor coverage. A similar argument can be made for the case when the sensor is one or more laser scanners.

Problem 2 (CUSP): Given $N$ UGVs and a polyhedral area $A$, find a set of waypoint-paths $P=\left\{P^{1}, \ldots, P^{N}\right\}$ that solve the following optimization problem

$$
\begin{array}{cl}
\min _{P} & \sum_{j=1}^{n-1} \sum_{i=1}^{N}\left\|p_{j}^{i}-p_{(j+1)}^{i}\right\| \\
\text { such that } & \cup_{i} P^{i} \text { guards } A \\
& \mathcal{G}_{P(j)} \in \mathcal{C}, \forall j
\end{array}
$$

Here $P(j)=\left\{p_{j}^{1}, \ldots, p_{j}^{N}\right\}$ denotes the UGV positions at time instance $j$ and $\mathcal{G}_{P(j)}$ is the induced information graph when the UGVs are at $P(j)$. Further, $\mathcal{C}$ is the set of connected graphs on $N$ vertices, $P^{i}=\left\{p_{1}^{i}, \ldots, p_{n}^{i}\right\}, \mathbb{Z}_{N}^{+}=\{1, \ldots, N\}$ and the start and finish positions, denoted by $p_{1}^{i}, p_{n}^{i}, i \in \mathbb{Z}_{N}^{+}$ may be given.

\section{$\mathcal{N P \text { -hardness }}$}

In the following, it is shown that both MTUSP and CUSP are $\mathcal{N} \mathcal{P}$-hard. This implies that we can not hope to solve an arbitrary problem instances to optimality in reasonable time but must adopt heuristic solution methods. This is the topic of Section V.
Proposition 1: Problem 1 (MTUSP) is $\mathcal{N} \mathcal{P}$-hard.

Proof: The proof will build upon a polynomial reduction [16] from an arbitrary instance of a well-known $\mathcal{N} \mathcal{P}$ hard problem, namely the Euclidean-TSP (ETSP) [17], to a special instance of MTUSP.

Given an ETSP instance, $\left(n,\left[d_{i j}\right]\right)$, where $n$ is the number of cities and $\left[d_{i j}\right]$ denotes the matrix with inter-city distances, we are free to choose the following parts of Problem 1 (MTUSP) such that the achieved optimal solution corresponds to that of the given ETSP:

1) Number of UGVs, $N$.

2) Start and finish depots for all UGVs, $p_{1}^{i}, p_{n+1}^{i}, i \in \mathcal{Z}_{N}^{+}$.

3) Obstacle configuration.

4) Area to be surveyed, $A$.

5) Maximal sensor range, $R$.

Regarding the number of UGVs, $N=1$ is a natural selection. In order to achieve a tour for this single UGV, we locate the start and finish depot, $p_{1}^{1}, p_{n+1}^{1}$ at an arbitrary city cite, as long as they are set equal. Further, an obstacle-free environment is chosen and the area $A$ is taken as the union of isolated points located at the $n$ cites. Finally, we set $R=0$.

Due to these choices, the area $A$ is fully guarded if and only if the UGV visits all the city locations and since the distances are preserved, the optimal solution of this specially designed instance of the MTUSP will coincide with the optimal solution of the given ETSP. This completes the proof.

\section{Proposition 2: Problem 2 (CUSP) is $\mathcal{N} \mathcal{P}$-hard.}

Proof: Follows directly from the previous proof since in the single UGV case, the connectivity constraint is trivially fulfilled.

\section{Proposed Solution}

In this section we will propose solutions to Problem 1 (MTUSP) and Problem 2 (CUSP) respectively.

\section{A. MTUSP}

As for the MTUSP, the solution encompasses three subproblems, as illustrated in Figure 2. We begin by formally stating the algorithm, and then describe the subproblems in somewhat more detail.

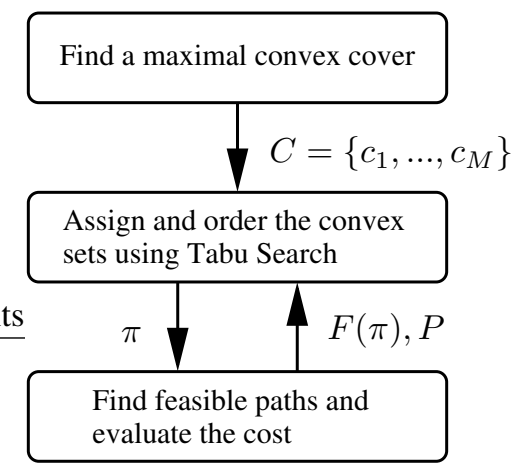

Fig. 2. The proposed solution relies on decomposing the problem into three subproblems. 
Algorithm 1 (Proposed solution for MTUSP): The algorithm consists of the following two steps, where the second step involves the iterative solution of two subproblems:

1) Create a maximal convex cover $C=\left\{c_{1}, \ldots, c_{M}\right\}$ of area $A$ in accordance with Algorithm 2 in [1].

2) Solve the following combined assignment and ordering problem using Tabu search:

$$
\min _{\pi} F(\pi)=\alpha \max _{i} f_{i}(\pi)+(1-\alpha) \Sigma_{i} f_{i}(\pi)
$$

where $\pi$ is a permutation of $\mathcal{Z}_{M+N}^{+}$, representing the assignment/ordering of the $M$ convex sets to the $N$ UGVs, $\alpha \in[01]$, and $f_{i}(\pi)$ is the optimal path length of UGV $i$ given the constraints in $\pi$. The value of $f_{i}(\pi)$ is found in a sub-routine by using a shortest path formulation to solve the following optimization problem:

$$
\begin{aligned}
f_{i}(\pi)=\min _{P^{i}} & \Sigma_{j}\left\|p_{j}^{i}-p_{(j+1)}^{i}\right\| \\
\text { s.t. } & P^{i} \text { guards } \cup_{I_{i}^{\pi}} c_{j} \\
& P^{i} \text { visits } c_{I_{i}^{\pi}(j)} \text { before } c_{I_{i}^{\pi}(j+1)}, j \in \mathcal{Z}_{\left|I_{i}^{\pi}\right|-1}^{+}
\end{aligned}
$$

Here, $I_{i}^{\pi}$ is the index of the sets in $C$ that are assigned to UGV $i$ in the minimization of $F$. In (1), $\alpha=1$ corresponds to the minimum time problem (MTUSP) and $\alpha=0$ corresponds to the minimum distance problem. Examples of both these options are found in Figure 6 in Section VI.

In the first subproblem, the computationally intractable problem of finding the minimum time paths that enable complete regional surveillance, is turned into a finite dimensional combinatorial optimization problem. This is achieved by finding a maximal convex cover of $A$, as defined in Section III. In the second subproblem, the order in which to visit the sets in the cover is determined using Tabu search. The third subproblem, which is called as a subroutine of the second one to evaluate the objective function in the Tabu search, involves a shortest path problem on a special graph, constructed from the given visitation order. Most of the details of the implementation of these three subproblems are identical to those presented in [1]. Hence, the reader is referred to that paper for a fuller description.

Below we will show that Algorithm 1 does result in a complete coverage, i.e., it solves Problem 1. Before doing so we first note that decomposing the problem into subproblems, might remove the optimal solution from the new set of feasible solutions. However, since it was shown in Section IV that Problem 1 (MTUSP) is $\mathcal{N} \mathcal{P}$-hard, our aim is not to solve the problem to optimality, but rather to produce good-enough solutions in reasonable time.

Proposition 3: Algorithm 1 produces a feasible solution to Problem 1 (MTUSP).

Proof: This is clear from Lemma 1 and the following three observations regarding Algorithm 1:

1) A convex cover is created.

2) All sets are assigned to different UGVs in (1).

3) Paths visiting all assigned sets are created in (2).
We now move on to the connectivity constrained version of the problem.

\section{B. CUSP}

The solution method suggested for Problem 2 (CUSP), is tailored for handling the hard connectivity constraints explicitly and builds upon the notion of a connectivityprimitive; introduced in Definition 6 and depicted in Figure 3. As depicted in Figure 4, the suggested algorithm for

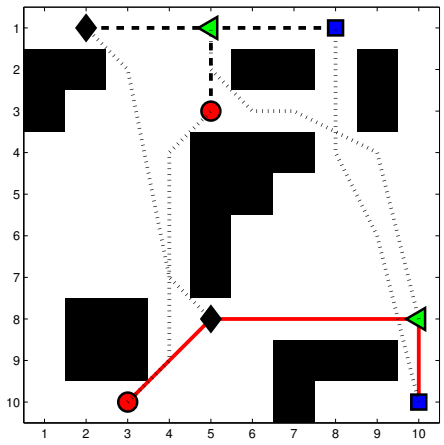

Fig. 3. Two connectivity-primitives (in solid and dashed) composed of four UGV positions each. The connectivity-primitives are created in step two of the algorithm. The dotted paths indicate the result of step three, i.e., how the UGVs should move from one primitive to another. The sum of these path-lengths is then used as distance $d_{i j}$ between the two primitives when solving the TSP in step four to find the order in which to visit the primitives.

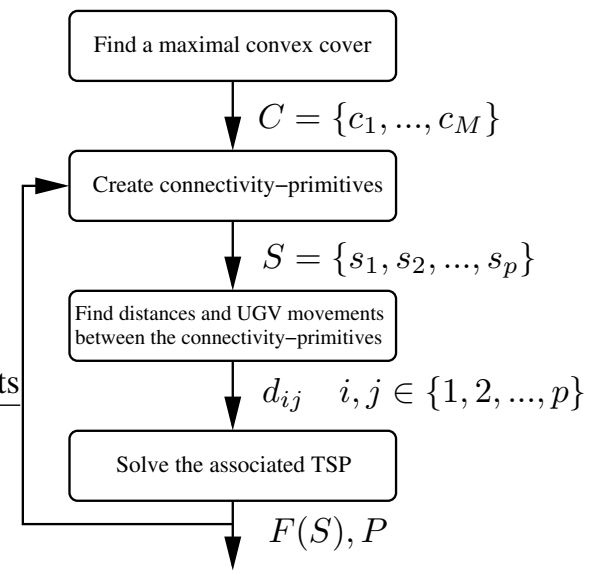

Fig. 4. Schematic overview of the suggested algorithm for solving CUSP

solving CUSP consists of four subproblems. We first state the algorithm and then discuss the four steps in detail.

Algorithm 2 (Proposed solution for CUSP): The algorithm consists of the following four steps:

1) Create a maximal convex cover $C=\left\{c_{1}, \ldots, c_{M}\right\}$ of area $A$ in accordance with Algorithm 2 in [1].

2) Create a set of connectivity-primitives, $S=$ $\left\{s_{1}, s_{2}, \ldots, s_{p}\right\}$, that completely covers the area, i.e., the convex cover $C$ is visited by the path $\cup_{i} s_{i}$.

3) Solve an assignment problem for each pair $\left(s_{i}, s_{j}\right)$ of connectivity-primitives in $S$, where the cost of an 
assignment of $p_{k} \in s_{i}$ to $p_{l} \in s_{j}$ is equal to the shortest obstacle free path from $p_{k}$ to $p_{l}$. Let $d_{i j}$ be the sum of these path-lengths in the optimal assignment.

4) Solve a TSP having the $p$ connectivity-primitives in $S$ as cities and the total path lengths $d_{i j}$ as inter city distances. While the maximal number of iterations have not been reached, goto 2 .

The first subproblem is the same as in Algorithm 1, finding a maximal convex cover.

In the second subproblem, a collection of connectivityprimitives, $S=\left\{s_{1}, s_{2}, \ldots, s_{p}\right\}$, is created in such a way that the entire area is covered, i.e., there is at least one connectivity-primitive node in every set of the cover, $C$. Let $V \subseteq C$ be the set of convex sets that have been visited by a connectivity-primitive already. In order to keep the number of connectivity-primitives low, it is preferable that the next connectivity-primitive is created in the set $C \backslash V$. This procedure is repeated until $C \backslash V=\varnothing$ which means that by visiting the connectivity-primitives, $S=\left\{s_{1}, s_{2}, \ldots, s_{p}\right\}$, the entire area is surveyed.

In order to decide in which order to visit the primitives (subproblem four) we need some notion of how far apart they are. In the third subproblem we find such distances $d_{i j}$ by solving an assignment problem for each pair of connectivity-primitives. One such assignment is depicted in Figure 3. We let the distance be the minimal combined length all UGVs need to travel to get from primitive $i$ to primitive $j$. The $\frac{p(p-1)}{2}$ assignment problems as well as the underlying shortest path problem are solved by polynomial time algorithms, [16].

The distances between the connectivity-primitives, denoted $d_{i j}$ in Figure 4, are then passed down to the fourth and last subproblem which is to determine the order in which to visit the connectivity-primitives as well as the objective function, $F(S)$ equal to total distance traveled by all UGVs. This is in fact a Traveling Salesmen Problem (TSP) where connectivity-primitives play the role of cities and $d_{i j}$ the corresponding distances. This also explains our earlier concern to keep the number of connectivity-primitives low in subproblem two. Various heuristic solution methods exist for solving TSPs [17]. In our particular case, a Simulated Annealing algorithm has been adopted. This then generates a feasible solution to the CUSP and the algorithm may stop. However, in order to get closer to an optimal solution, it may be beneficial to re-do the process for a particular number of iterations by repeating the last three subproblems.

Proposition 4: Algorithm 2 produces a feasible solution to Problem 2 (CUSP).

Proof: This is since all connectivity-primitives are visited and they are designed to both respect the connectivity constraints and provide a complete coverage of area, $A$.

Having discussed the algorithms in detail, it is now time to run some simulation examples.

\section{Simulations}

In this section, a small selection of the simulations made is presented. The objective is to highlight some of the key characteristics of the proposed solution method. Throughout this section, the search area $A$ is chosen to be all of the obstacle free space, i.e., the white area in all figures.

\section{A. MTUSP simulations}

In all figures of this section, the initial position of the UGVs are marked with a square (ם), while the final positions are marked with a diamond $(\downarrow)$. These two, together with the filled larger circles represent the surveillance points for guarding $A$.

The first simulation, found in Figure 5, illustrates the cooperative nature of the MTUSP. The final positions of the UGVs are here free variables to be chosen by the optimization routine. As can be seen, this extra degree of freedom is used constructively so that the UGVs survey the horizontally and vertically aligned streets in a cooperative manner with the common objective of minimizing the search time, i.e., we have chosen $\alpha=1$ in (1).

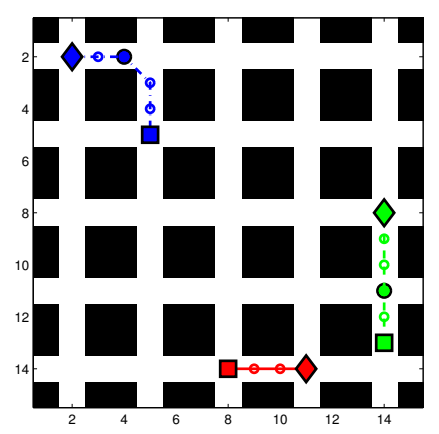

Fig. 5. The Manhattan grid is surveyed cooperatively in minimum time. The starting points of the UGVs are marked with $\mathbf{\square}$.

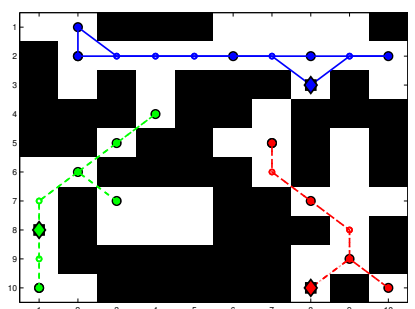

(a) Minimum time objective.

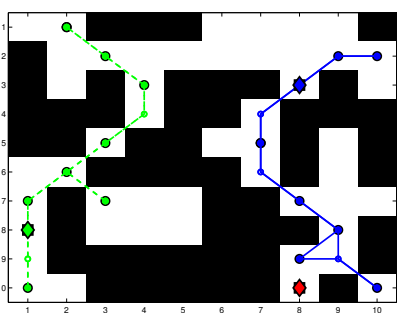

(b) Minimum distance objective.
Fig. 6. Two MTUSP simulation examples with different objectives.

Figure 6 illuminates the interplay between the choice of the objective function and the obtained solutions. In Figure 6(a), the solutions are obtained by minimizing the total search time. It can be noted that these solutions distribute the work load quite evenly over the vehicle fleet. In Figure 6(b) however, the objective has been set to minimize the total distance traveled by the UGVs, i.e., $\alpha=0$ in (1). Since this option does not take into consideration the division of the work load between the different vehicles, the resulting solutions often do not utilize some of the vehicles at all. This may be of interest e.g. when unemployed vehicles can be used to perform other tasks. 


\section{B. CUSP simulations}

In Figure 7, the starting positions of the UGVs are chosen randomly while the final positions are optimized by Algorithm 2. The most important aspect to notice is that the three UGVs are not restricted to pass on the same "side" of the obstacles but are nevertheless recurrently connected at the five surveillance instances, Figure 7(b)- 7(f). Also, notice that the randomly selected initial positions in Figure 7(a) do not necessarily induce a connected information graph and that the area is completely surveyed.

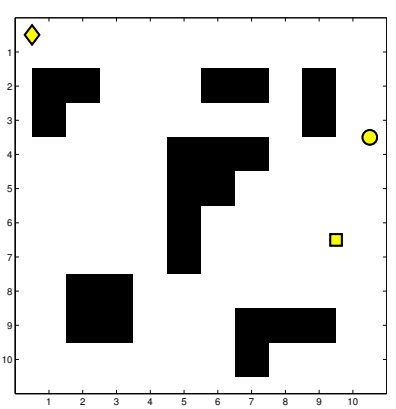

(a)

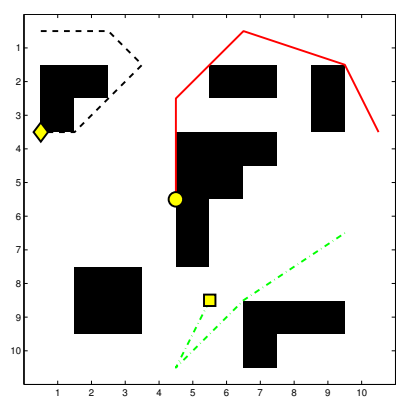

(c)

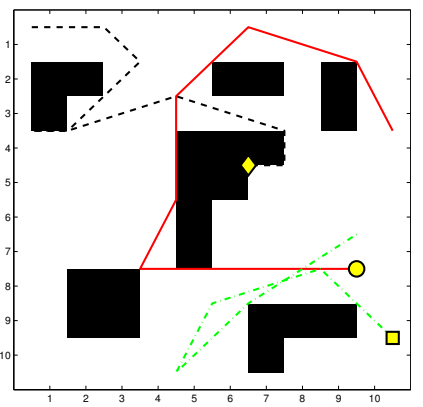

(e)

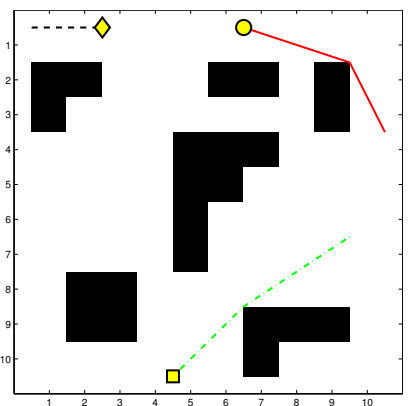

(b)

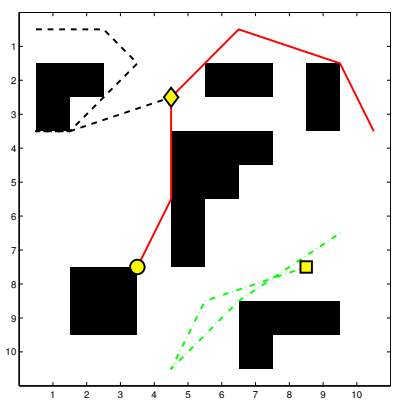

(d)

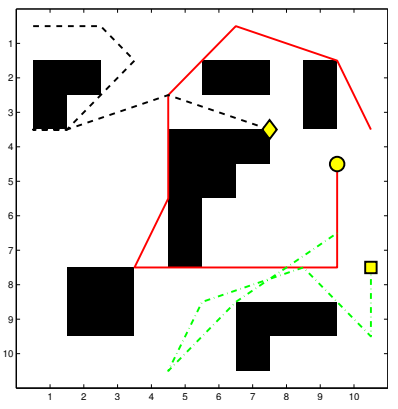

(f)
Fig. 7. An example CUSP simulation. The UGVs are recurrently connected at the five surveillance instances in Figure 7(b)- 7(f). Notice how they are free to pass on different "sides" of the obstacles and that the randomly selected initial positions in Figure 7(a) do not necessarily induce a connected information graph.

\section{CONCLUDING REMARKS}

Problem 1 (MTUSP), which is the basic problem of this paper, considers minimum-time surveillance of a given polyhedral area with obstacles using a group of UGVs. The on-board sensors are assumed to be occluded by the obstacles and limited by a maximal sensor range. In Problem 2 (CUSP) we also impose connectivity constraints by requiring the induced information graph to be recurrently connected. As a distinguishing feature, the considered communication restrictions may involve both line-of-sight constraints and limited sensor range constraints in the presence of obstacles. The main motivation for introducing this weaker notion of connectivity is surveillance applications where the sentry vehicles may have to split temporary in order to complete the given mission efficiently but are required to establish contact recurrently in order to exchange information and/or to make sure that all units are intact and well-functioning.

\section{REFERENCES}

[1] Anisi, D. A. and Ögren, P., "Minimum Time Multi-UGV Surveillance," Optimization and Cooperative Control Strategies, edited by M. Hirsch, C. Commander, P. Pardalos, and R. Murphey, Lecture Notes in Control and Information Sciences, Springer Verlag, 2008.

[2] Anisi, D. A., Ögren, P., and Hu, X., "Communication constrained multi-UGV surveillance," Proc. of the $17^{\text {th }}$ IFAC World Congress, Seoul, South Korea, 6-11 Jul. 2008.

[3] Acar, E., Choset, H., Zhang, Y., and Schervish, M., "Path Planning for Robotic Demining: Robust Sensor-based Coverage of Unstructured Environments and Probabilistic Methods." International Journal of Robotics Research, Vol. 22, No. 7, 2003, pp. 441-466.

[4] Choset, H., "Coverage for Robotics-A Survey of Recent Results," Annals of Mathematics and Artificial Intelligence, Vol. 31, No. 1, 2001, pp. 113-126.

[5] Ge, S. and Fua, C., "Complete Multi-Robot Coverage of Unknown Environments with Minimum Repeated Coverage," Proceedings of the IEEE International Conference on Robotics and Automation, 2005.

[6] Carroll, D., Everett, H., Gilbreath, G., and Mullens, K., "Extending Mobile Security Robots to Force Protection Missions," AUVSI Unmanned Systems, 2002, pp. 9-11.

[7] Ablavsky, V., Stouch, D., and Snorrason, M., "Search Path Optimization for UAVs Using Stochastic Sampling With Abstract Pattern Descriptors," Proceedings of the AIAA Guidance Navigation and Control Conference, Austin, TX, August, 2003.

[8] John, M., Panton, D., and White, K., "Mission Planning for Regional Surveillance," Annals of Operations Research, Vol. 108, 2001, pp. 157-173.

[9] Hespanha, J., Kim, H., and Sastry, S., "Multiple-Agent Probabilistic Pursuit-Evasion Games," Decision and Control, 1999. Proceedings of the 38th IEEE Conference on, Phoenix, AZ, Vol. 3, 1999, pp. 24322437.

[10] Nilsson, U., Ögren, P., and Thunberg, J., "Optimal Positioning of Surveillance UGVs," IEEE Conference on Intelligent Robots and Systems (IROS), 2008.

[11] Urrutia, J., "Art Gallery and Illumination Problems," Handbook of computational geometry, edited by J.-R. Sack and J. Urrutia, NorthHolland Publishing Co., 2000, pp. 973-1027.

[12] Gerkey, B., Thrun, S., and Gordon, G., "Visibility-Based PursuitEvasion with Limited Field of View," The International Journal of Robotics Research, Vol. 25, No. 4, 2006, pp. 299-315.

[13] Burgard, W., Moors, M., Fox, D., Simmons, R., and Thrun, S., "Collaborative Multi-Robot Exploration," IEEE International Conference on Robotics and Automation (ICRA), Vol. 1, 2000.

[14] Schouwenaars, T., Feron, E., and How, J., "Multi-vehicle path planning for non-line of sight communication," Proc. of the 2006 American Control Conference (ACC), Minneapolis, Minnesota, USA, Jun. 2006, pp. $5757-5762$.

[15] Esposito, J. M. and Dunbar, T. W., "Maintaining wireless connectivity constraints for swarms in the presence of obstacles," Proc. of the 2006 IEEE International Conference on Robotics and Automation (ICRA), Orlando, Florida, May 2006, pp. 946-951.

[16] Papadimitriou, C. H. and Steiglitz, K., Combinatorial optimization: algorithms and complexity, Dover Publications Inc., Mineola, NY, 1998.

[17] Bektas, T., "The multiple traveling salesman problem: an overview of formulations and solution procedures," Omega, Vol. 34, No. 3, Jun. 2006, pp. 209-219. 